\title{
ACOMPANHAMENTO DA PRESCRIÇÃO DE FÁRMACOS DE USO HUMANO PARA FINS DE TERAPIA ANIMAL
}

\author{
Daniel Andrade da Silva; Maria Eduarda Canôa Bertão; Willian Marinho Dourado Coelho \\ ${ }^{1}$ Fundação Eduucacional de Andradina - FEA, Faculdades de Ciências Agrárias de Andradina - FCAA, Curso de Medicina \\ Veterinária, Andradina, SP. E- mail: silvaparasempre_123@hotmail.com
}

\begin{abstract}
RESUMO
O objetivo deste estudo foi aferir quantitativamente a prescrição de medicamento humano, para terapia animal. Desenvolveu- se em 14 estabelecimentos comercias que se destina a venda de medicamentos humanos, em três cidades do interior paulista e duas cidades Sul Mato-grossenses, teve inicio em 10 de abril de 2017 perdurando até 30 de setembro de 2017, foi entregue uma ficha onde deveria ser transcrita toda medicação prescrita por um Médico Veterinário, estas foram coletadas uma vez ao mês e auditadas perante sua classificação. Durante o estudo foram prescrevidos 1.034 medicamentos, dentre estes, antimicrobianos somaram 45,5\% dos fármacos prescritos, seguido pelo corticosteróide 15,38\%, vitaminas e minerais 10,45 \%, diuréticos e psicotrópicos $8,03 \%$, anti- eméticos 5,61\%, anti- inflamatório não esteroide e protetores gástricos com $2,41 \%$ cada, anti- hipertensivo $1,45 \%$ e anti- histamínicos com $0,68 \%$. Os dados mostram que a prescrição de drogas fabricadas para uso humano é de suma importância.
\end{abstract}

Palavras- chave: antimicrobiano; corticosteróide; drogas; medicação; psicotrópicos.

\section{MONITORING THE PRESCRIPTION OF HUMAN USE DRUGS FOR ANIMAL THERAPY PURPOSES}

\begin{abstract}
The objective of this study was to quantitatively measure the human drug prescription for animal therapy. It was developed in 14 commercial establishments for the sale of human medicines, in three cities in the interior of São Paulo and two cities in the south of Mato Grosso, started on April 1, 2017 and lasted until September 30, 2017, a record was issued where all medication prescribed by a Veterinarian should be transcribed, these were collected once a month and audited before being classified. During the study, 1,034 medications were prescribed, among them antimicrobials added $45.5 \%$ of the prescribed drugs, followed by corticosteroids $15.38 \%$, vitamins and minerals $10.45 \%$, diuretics and psychotropic drugs $8.03 \%$, antiemetics 5, 61\%, non-steroidal antiinflammatory and gastric protectors with $2.41 \%$ each, antihypertensive $1.45 \%$ and antihistamines with $0.68 \%$. The data show that the prescription of drugs manufactured for human use is of paramount importance.
\end{abstract}

Key words: antimicrobial; corticosteroid; drugs; medication; psychotropics.

\section{INTRODUÇÃO}

A prescrição na Medicina Veterinária compete na escolha do medicamento que mais se adeque a disfunção fisiológica do paciente, considerando seu diagnóstico, prognóstico e custo benefício, sendo assim, torna- se necessário o conhecimento da vasta variedade de opções terapêuticas a disposição no mercado (MILLER, 1977; ADAMS, 1995), respeitando princípios éticos de bem estar da sociedade e dos animais (ESTOL, 2001). Alguns fármacos não são fabricados 
exclusivamente na indústria veterinária, assim, o Médico Veterinário prescreve drogas licenciadas para uso humano, a fim de um tratamento eficaz de seu paciente (CRMV- ES s/d).

O objetivo deste estudo foi aferir quantitativamente a prescrição de medicamento humano, para fins de terapia animal em 14 farmácias, distribuídas nas cidades de Andradina- SP, Castilho- SP, Inocência- MS, Nova independência- SP e Três Lagoas- MS.

\section{METODOLOGIA}

O estudo foi desenvolvido em 14 (quatorze) estabelecimentos comercias que se destina a venda de medicamentos humanos, em três cidades do interior paulista (Andradina, Castilho e Nova Independência) e duas cidades Sul Mato-grossenses (Inocência e Três Lagoas), que aceitaram participar do experimento. Teve inicio em 10 de abril de 2017 perdurando até 30 (trinta) de setembro do mesmo ano. Foi entregue uma ficha identificada com nome da farmácia, cidade, endereço e mês, onde deveria ser transcrita toda e qualquer medicação prescrita por um Médico Veterinário (Fig. 1), estas informações foram coletadas uma vez ao mês, e auditadas perante sua classificação farmacológica.

Figura 1. Ficha mensal confeccionada para o estudo

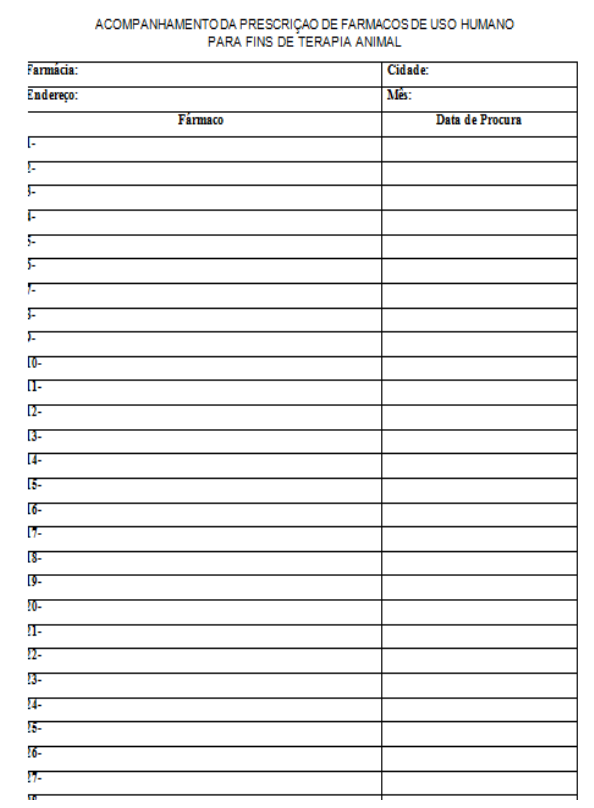

\section{RESULTADOS}

Durante todo o estudo foram prescrevidos 1.034 (um mil e trinta e quatro) medicamentos. Dentre estes os antimicrobianos somaram 45,5\% (471/1.034) dos fármacos prescritos, seguido pelos corticosteróides com 15,38\% (159/ 1.034), vitaminas e minerais 10,45 \% (108 / 1.034), diuréticos e psicotrópicos com 8,03\% (83/ 1.034), anti- eméticos 5,61\% (58/ 1.034), antiinflamatório não esteroide e protetores gástricos 2,41\% (25/ 1.034), anti- hipertensivo 1,45 \% (15/ 1.034) e os anti- histamínicos $0,68 \%$ (7/ 1.034) (Tab. 1 e Fig. 2). Maio foi o mês com a maior procura por fármacos $19,92 \%$ (206/ 1.034), por outro lado, o mês subsequente junho trouxe a menor procura com 13, $64 \%$ (141/ 1.034). 
Tabela 1. Medicamentos prescritos de acordo com sua classificação farmacológica.

\begin{tabular}{llllllll} 
& Abril & Maio & Junho & Julho & Agosto & \multicolumn{2}{c}{ Setembro TOTAL } \\
\cline { 2 - 8 } A. I. N. E. & 2 & 8 & 3 & 4 & 1 & 7 & 25 \\
\hline Anti- eméticos & 7 & 21 & 10 & 8 & 6 & 6 & 58 \\
\hline Anti- hipertensivo & 5 & 3 & 1 & 1 & 1 & 4 & 15 \\
\hline Anti- histamínico & 3 & 0 & 3 & 0 & 1 & 0 & 7 \\
\hline Antimicrobiano & 98 & 90 & 61 & 73 & 71 & 78 & 471 \\
\hline Corticosteróides & 35 & 29 & 21 & 29 & 19 & 26 & 159 \\
\hline Diuréticos & 14 & 21 & 17 & 8 & 10 & 13 & 83 \\
\hline Protetores gástrico & 1 & 5 & 1 & 10 & 5 & 3 & 25 \\
\hline Psicotrópicos & 11 & 12 & 13 & 11 & 15 & 21 & 83 \\
\hline Vitaminas e minerais & 28 & 17 & 11 & 17 & 13 & 22 & 108 \\
\hline TOTAL & 204 & 206 & 141 & 161 & 142 & 180 & 1.034 \\
\hline
\end{tabular}

Figura 2. Procura de fármaco referente ao mês.

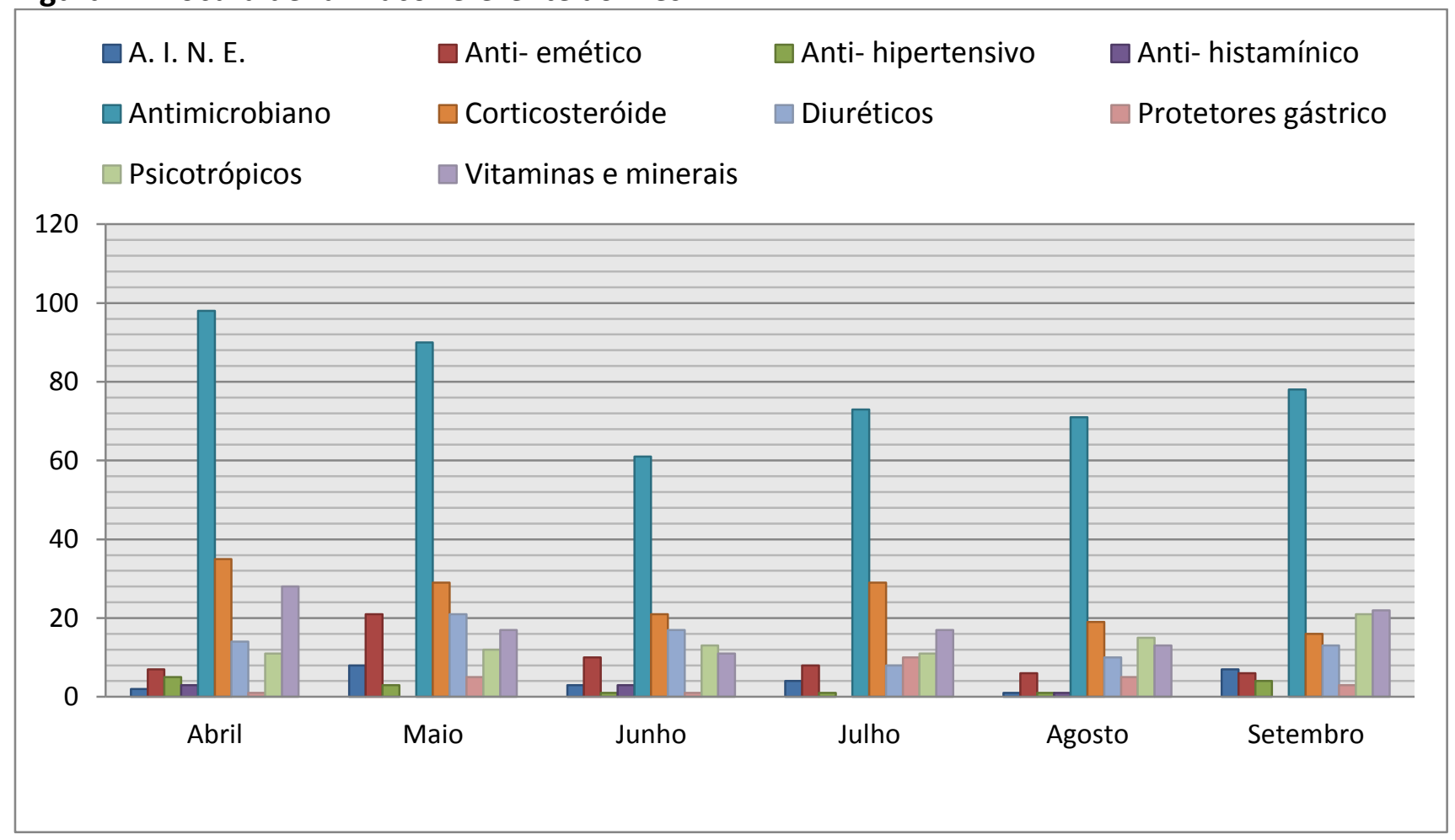

\section{DISCUSSÃO}

O elevado indice de prescrições de antimicrobianos de uso humano encontrado em nosso estudo, condis com a RDC no 20/2011 que não proíbe a prescrição desta classe de droga desde que, seja redigido um receituário em duas vias contendo identificação do emitente, dados pertinentes ao proprietário (nome, endereço e Registro Geral) e paciente (nome, espécie, raça e porte), sendo que, se fazem dispensáveis o preenchimento dos campos destinados á idade e sexo do animal, uma vez que estes dados não somam ao monitoramento farmacoepidemiológico (ANVISA, 2013), desta maneira, é comum uso de antimicrobianos licenciados pra venda em 
estabelecimento humano para terapia animal, devido à falta de opção no mercado de medicamentos veterinários (SILVEIRA, 2011).

As classes de antimicrobianos mais utilizados na medicina veterinária são: aminoglicosídeos, cefalosporinas, lincosamidas, macrolídeos, penicilinas, sulfonamidas e tetraciclinas (BAHR ARIAS et al., 2008), encontrando- se com melhor aplicabilidade em drogarias humanas, como visto em nosso experimento, sendo que, estas classes de antibióticos prescritos para animais, pertencem as mesmas das empregadas aos seres humanos (SCHWARZ et al., 2001).

Os psicotrópicos foram uma das classes farmacológicas mais relatadas em nosso trabalho, pelo fato de que o Médico Veterinário, para possuir um estoque destes em seu estabelecimento, necessita de um espaço físico reservado ou armário, com fechadura que ficará sob guarda do responsável técnico (Médico Veterinário ou Farmacêutico) dentre outras exigências (ANVISA, 2010).

Algumas patologias na clínica de pequenos animais necessitam de uma terapia onde deve ser feita a associação de medicamentos, exemplificando a erliquiose, vulgarmente conhecida como doença do carrapato, que requer uma terapêutica simultânea de um antimicrobiano, no caso a Doxiciclina (DAVOUST, 1993) juntamente a corticoterapia para que haja a reversão do quadro de trombocitopenia (VIGNARD et al., 2001), o que condiz com a realidade da região onde o estudo foi realizado, devido as condições climáticas que favorecem a disseminação da enfermidade.

\section{CONCLUSÃO}

Os dados fornecidos pelo nosso estudo mostram que a prescrição de medicamentos de uso humano na Medicina Veterinária, é de suma importância para os animais e saúde pública, visto que, drogas utilizadas para tratamento de moléstias do homem, também são atribuídas na terapia animal e a grande relevância da medicação humana no caso dos psicotrópicos, por falta de alternativa comercial na farmácia veterinária, do mesmo modo algumas formulações de antimicrobianos.

\section{REFERÊNCIAS}

ADAMS, R. A. Veterinary Pharmacology and Therapeutics. $7^{\text {th }}$. Ed. Ames, lowa, USA: lowa State University Press, 1995. Cap. 1. p. 1141-2.

ANVISA. Referências técnicas para atividades veterinárias. REFERÊNCIA TÉCNICA PARA FUNCIONAMENTO DOS SERVIÇOS VETERINÁRIOS. Brasília 04 de Março de 2010. http://portal.anvisa.gov.br/documents/33856/2054354/Refer\%C3\%AAncia+t\%C3\%A9cnica+para+ o+funcionamento+dos+servi\%C3\%A7os+veterin\%C3\%A1rios/057287c3-823e-41aa-a121$\underline{672 \mathrm{~cd} 54 \mathrm{faaa} 8 .}$.

ANVISA. Orientações de procedimentos relativos ao controle de medicamentos à base de substâncias classificadas como antimicrobianos, de uso sob prescrição isoladas ou em associação. NOTA TÉCNICA SOBRE A RDC N 20/2011. Brasília 24 de Setembro de 2013. http://www.anvisa.gov.br/sngpc/documentos\%202013/Nota Tecnica RDC n 20201124092 013.pdf.

BAHR ARIAS, M. V. B.; BATAGLLIA, L. A.; AIELLO, G.; CARVALHO, T. T.; FREITAS, J. C. Identificação da suscetibilidade antimicrobiana de bactérias isoladas de cães e gatos com feridas traumáticas contaminadas e infectadas. Semina: Ciências Agrárias, Londrina, v. 29, n. 8, p, 861- 874, 2008. 
CRMV- ES. Orientações para prescrição de fármacos humanos utilizados no tratamento animal. s/d. crmves.org.br/crmv/assets/userfiles/files/receituario.pdf.

DAVOUST, B. - Canine ehrlichiosis, Point Vét., 25 (151): 43-51, 1993.

ESTOL, L. Educação Veterinária na América Latina no século XXI. Conferência internacional sobre interações animais, Setembro, 2001, Rio de Janeiro.

MILLER, L. C. Doctors, Drugs, and Names. Journal of the American Medical Association. v. 177, p. 97- 103, 1997.

SCHWARZ, S.; KEHRENBERG, C.; WALSH, T. R. Use of antimicrobial agent in veterinary medicine and food animal production. International Journal of Antimicrobial Agents. Amsterdam, v. 17, n. 6 , p. 431- 437, 2001. https://doi.org/10.1016/S0924-8579(01)00297-7

SILVEIRA, C. Nova regra para antibióticos também deve ser seguida por donos de pets. Globo.com. São Paulo. 2011. http://g1.globo.com/sao-paulo/noticia/2011/01/nova-regra-para-antibioticostambem-deve-ser-seguida-por-donos-de-pets.html

VIGNARD, R. K.; ALVES, F.R.; BLEICH, I. Erliquiose canina. Cães e Gatos, Sorocaba, n. 96, p. 25-28, 2001. 